\title{
BRAIN TUMOR MRI IMAGE SEGMENTATION AND DETECTION IN IMAGE PROCESSING
}

\author{
Rohini Paul Joseph ${ }^{1}$, C. Senthil Singh ${ }^{2}$, M.Manikandan ${ }^{3}$ \\ ${ }^{1}$ M.Tech Student, ${ }^{2}$ Associate Professor, Electronics and Communication Engineering, Toc H Institute of Science and \\ Technology, Kerala, India \\ ${ }^{3}$ Department of Electronics, Anna University, Chennai, India
}

\begin{abstract}
Image processing is an active research area in which medical image processing is a highly challenging field. Medical imaging techniques are used to image the inner portions of the human body for medical diagnosis. Brain tumor is a serious life altering disease condition. Image segmentation plays a significant role in image processing as it helps in the extraction of suspicious regions from the medical images. In this paper we have proposed segmentation of brain MRI image using $K$-means clustering algorithm followed by morphological filtering which avoids the misclustered regions that can inevitably be formed after segmentation of the brain MRI image for detection of tumor location.
\end{abstract}

Keywords: Image Segmentation, MRI, K-means clustering, Morphological filtering. $* * *$

\section{INTRODUCTION}

Information is conveyed through images. Image processing is a process where input image is processed to get output also as an image. Main aim of all image processing techniques is to recognize the image or object under consideration easier visually [1].All the images used in today's world are in the digital format. Medical images are images that show the physical attributes distribution. Medical imaging modalities as in MRI, CT scan mostly depend on computer technology to generate or display digital images of the internal organs of the human body which helps the doctors to visualize the inner portions of the body. CT scanner, Ultrasound and Magnetic Resonance Imaging took over conventional x-ray imaging, by allowing the doctors see the body's third dimension [21].

\subsection{Magnetic Resonance Imaging}

Protons and neutrons of the nucleus of an atom has an angular momentum which is known as a spin. These spins will cancel when the number of subatomic particles in a nucleus is even. Nuclei with odd number will have a resultant spin .This forms the basis of magnetic resonance imaging. A magnetic resonance imaging (MRI) scanner uses powerful magnets to polarise and excite hydrogen nuclei (single proton) in human tissue, which produces a signal that can be detected and it is encoded spatially, resulting in images of the body. The MRI machine emits radio frequency (RF) pulse that specifically binds only to hydrogen. The system sends the pulse to that specific area of the body that needs to be examined. Due to the $\mathrm{RF}$ pulse, protons in that area absorb the energy needed to make them spin in a different direction. This is meant by the resonance of MRI. The RF pulse makes the protons spin at the larmour frequency, in a specific direction. This frequency is found based on the particular tissue being imaged and the strength of the main magnetic field. MRI uses three electromagnetic fields: static field which is a very strong static magnetic field which polarizes the hydrogen nuclei; gradient field which is a weaker time-varying field used for spatial encoding; and a weak radio frequency field for manipulation of the hydrogen nuclei to produce measurable signals, which are collected through radio frequency antenna.

\subsection{Challenges}

The brain is the anterior most part of the central nervous system. Brain tumor is an intracranial solid neoplasm. Tumors are created by an abnormal and uncontrolled cell division in the brain. In this work, we have used axial view of the brain image (2D) from MRI scan because MRI scan is less harmful than CT brain scan. A patient is subjected to different diagnostic methods to determine the cause of the symptoms mentioned by him. Techniques like performing a biopsy, performing imaging, like taking a MRI or CT scan of the brain will be done. In biopsy, pathologists take a specimen of the brain tissue under consideration for checking the presence of tumor. A pathologist looks at the tissue cells under a microscope to check for presence of abnormality. Though biopsy will show the presence of tumor and its pathology, when doctors go for surgery, they must know the tumor extent and the exact location of tumor in the brain, which can be found by taking MRI scan of the patient as MRI doesn't involve the use of harmful radiations when compared to CT scan. Traditional method in hospitals is to segment the 
medical image under consideration, manually and this depends on how well the physician can perceive the image under consideration to get the required region extracted out, which is made difficult because of minute variations and resemblance between the original and affected biological part in the image. The shortage of radiologists and the large volume of MRI to be analyzed make these readings labor intensive and also cost expensive. It also depends on the expertise of the technician examining the images [10]. Estimates also indicate that between 10 and $30 \%$ of tumors are missed by the radiologists during the routine screening.

During the acquisition of medical images, there are possibilities that the medical image one gets might be degraded because of problems that can occur during the acquisition stage. So the original image may not be suitable for analysis. Image segmentation can be defined as the partition or segmentation of a digital image into similar regions with a main aim to simplify the image under consideration into something that is more meaningful and easier to analyze visually. Image segmentation is the main significant process in the majority of medical image analysis. Image segmentation methods can be classified as thresholding, region based, supervised and unsupervised classification techniques.

Various approaches have been carried out in the field of brain tumor detection. Sindhushree. K.S, et al[14] have developed a brain tumor segmentation method and validated segmentation on two dimensional MRI data. Also, detected tumors are represented in 3-Dimensional view. High pass filtering, histogram equalization, thresholding, morphological operations and segmentation using connected component labeling was carried out to detect tumor. The two dimensional extracted tumor images were reconstructed into three dimensional volumetric data and the volume of the tumor was also calculated. M.C. Jobin Christ and R.M.S. Parvathi[6] proposed a methodology that integrates $\mathrm{K}$ Means clustering with marker controlled watershed segmentation algorithm and integrates Fuzzy C Means clustering with marker controlled watershed segmentation algorithm separately for medical image segmentation. The proposed methodology is a two stage process. First K-means clustering (Fuzzy C Means) is used to get a primary segmentation of the input image, and secondly marker controlled watershed segmentation algorithm is applied to the primary segmentation to get the final segmented image.

P.Vasuda, S.Satheesh [12], proposed a technique to detect tumors from MR images using fuzzy clustering technique. This algorithm uses fuzzy C-means but the major drawback of this algorithm is the computational time required. Classifiers are also known as supervised methods since they require training data that are manually segmented and then used as references for automatically segmenting new data. The use of the same training data for classifying a large number of images, may lead to biased result. Supervised segmentation method requires considerable amount of training and testing data which comparatively complicates the process[13].

\section{PROPOSED METHODOLOGY}

We have proposed segmentation of the brain MRI images for detection of tumors using clustering techniques. A cluster can be defined as a group of pixels where all the pixels in certain group defined by a similar relationship [1]. Clustering is also known as unsupervised classification technique. The name unsupervised classification because the algorithm automatically classifies objects based on user given criteria. Here K-means clustering algorithm for segmentation of the image followed by morphological filtering is used for tumor detection from the brain MRI images. The proposed block diagram is as shown.

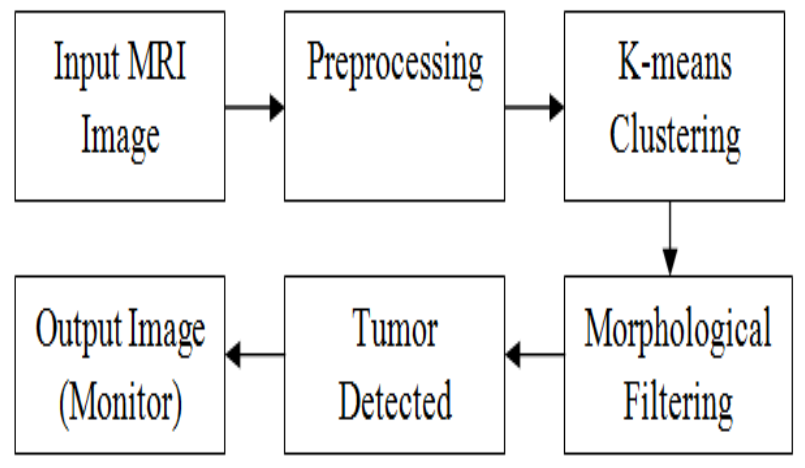

Fig-1: Proposed block diagram

MRI scans of the human brain forms the input images for our system where the grey scale MRI input images are given as the input. The preprocessing stage will convert the RGB input image to grey scale. Noise present if any, will be removed using a median filter. The preprocessed image is given for image segmentation using K-means clustering algorithm. As there are chances of occurrence of misclustered regions after the application of K-means clustering algorithm[15], we have proposed morphological filtering which is performed after the image is segmented by $\mathrm{K}$-means clustering algorithm.

\subsection{Proposed Algorithm}

The algorithm that we have proposed is as follows:

1. Let $\mathrm{x}_{1}, \ldots, \mathrm{x}_{\mathrm{M}}$ are $\mathrm{N}$ data points in the input image, let $\mathrm{k}$ be the number of clusters which is given by the user.

2. Choose $c_{1}, \ldots, c_{K}$ cluster centres.

3. Distance between each pixel and each cluster centre is found.

4. The distance function is given by 
$J=\left|x_{i}-c_{j}\right|$ for $i=1, \ldots, N$ and for $j=1, \ldots, k$, where $\quad \mid$ $\mathrm{x}_{\mathrm{i}}-\mathrm{c}_{\mathrm{H}}$, the absolute difference of the distance between a data point $x_{i}$ and the cluster centre $c_{3}$ indicates the distance of the $\mathrm{N}$ data points from their respective cluster centers.

5. Distribute the datapoints $\mathrm{x}$ among the $\mathrm{k}$ clusters using the relation

$x \in C_{i}$ if $\left|x-c_{j}\right|<\left|x-c_{j}\right|$ for $i=1,2, \ldots, k, i \neq j$, where $C_{j}$ denotes the set of data points whose cluster centre is $c_{\mathrm{j}}$.

6. Updated cluster centre is given as, $c_{i}=\frac{1}{m_{i}} \Sigma_{k=C_{i}} x$, for $\mathrm{i}=1, \ldots, k$, where $m_{i}$ is the number of objects in the dataset $C_{i}$, where $C_{i}$ is the $i^{\text {th }}$ cluster and $\mathrm{c}_{\mathrm{i}}$ is the centre of cluster $\mathrm{C}_{\mathbf{i}}$.

7. Repeat from Step 5 to Step 8 till convergence is met.

8. After segmentation and detection of the desired region, there are chances for misclustered regions to occur after the segmentation algorithm, hence morphological filtering is performed for enhancement of the tumor detected portion. Here structuring element used is disk shaped.

\subsection{Morphological Filtering}

Morphology is the study of shapes and structures from a scientific perspective. Morphological filters are formed from the basic morphology operations. A structuring element is mainly required for any morphological operation. Morphological operations operate on two images, structuring element and the input image. Structuring elements are small images that are used to probe an input image for properties of interest. Origin of a structuring element is defined by the centre pixel of the structuring element. In morphology, the structuring element defined will pass over a section of the input image where this section is defined by the neighbourhood window of the structuring element and the structuring element either fits or not fits the input image. Wherever the fit takes place, corresponding image that represents the input image's structure is got and suppression of the geometric features of the input image that doesn't fit the structuring element's neighbourhood takes place. Two main morphology operations are erosion and dilation where erosion results in the thinning of the objects in the image considered and dilation results in thickening of the objects in the image. Dilation uses the highest value of all the pixels in the neighbourhood of the input image defined by the structuring element and erosion uses the lowest value of all the pixels in the neighbourhood of the input image.

\section{SIMULATION RESULTS}

Some of the brain MR images containing tumor taken for testing our proposed algorithm are shown.
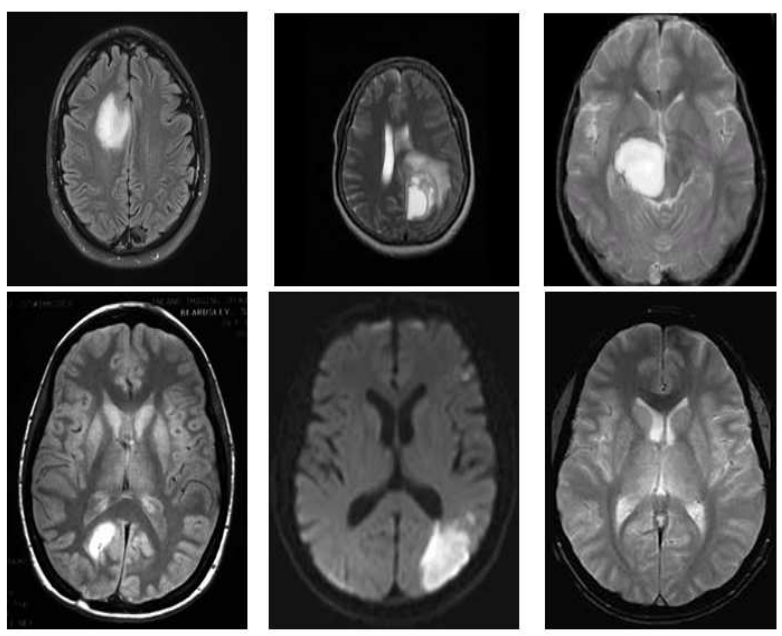

Fig-2: Brain MR images containing tumor

The brain tumor location is found out by applying our proposed algorithm using Matlab Simulator. A GUI (Graphical User Interface) is created to make the system userfriendly. Collect the required input brain MR image from the database which is shown in Fig 2. In our design we have taken the number of clusters as four. Fig 4 shows the final clustering of brain MR image after being processed by our algorithm. Fig 5 shows the final tumor detected portion from brain MR image.

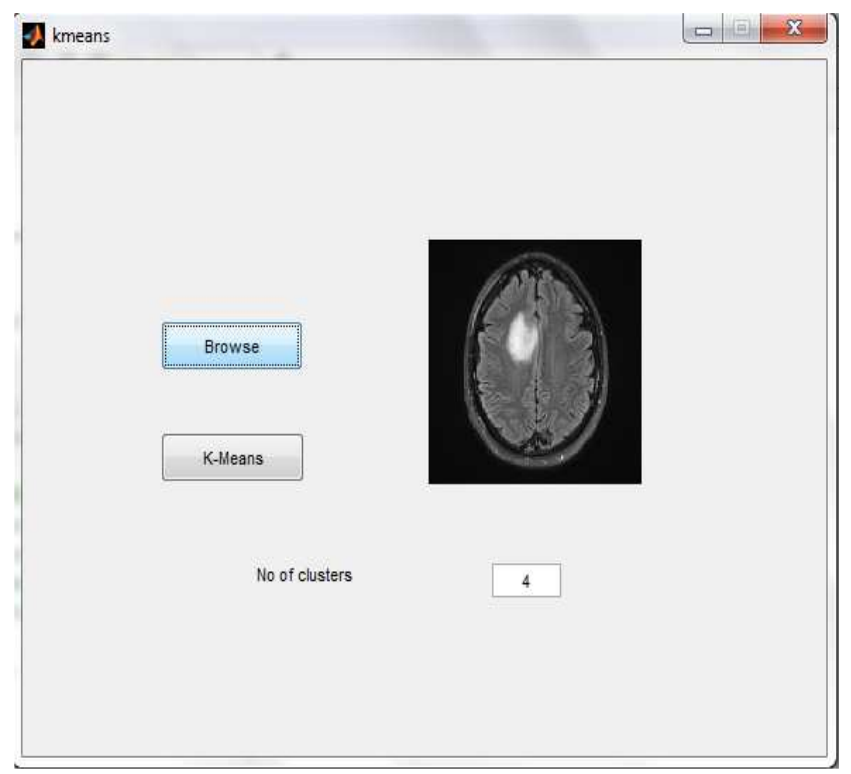

Fig- 3: Original brain MR image with tumor 


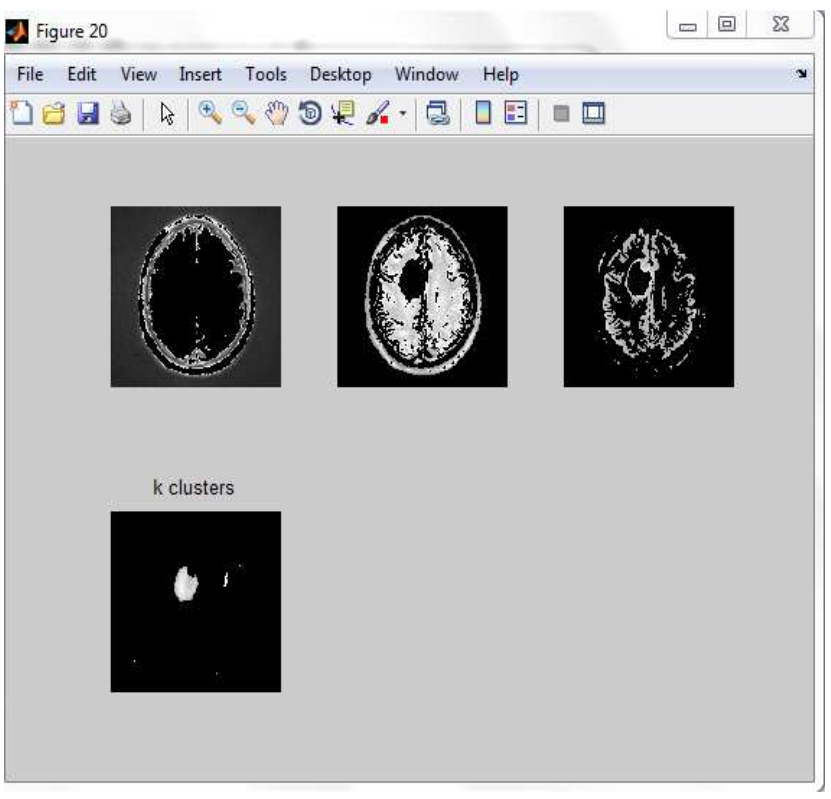

Fig-4: Clustering of brain tumor MR image

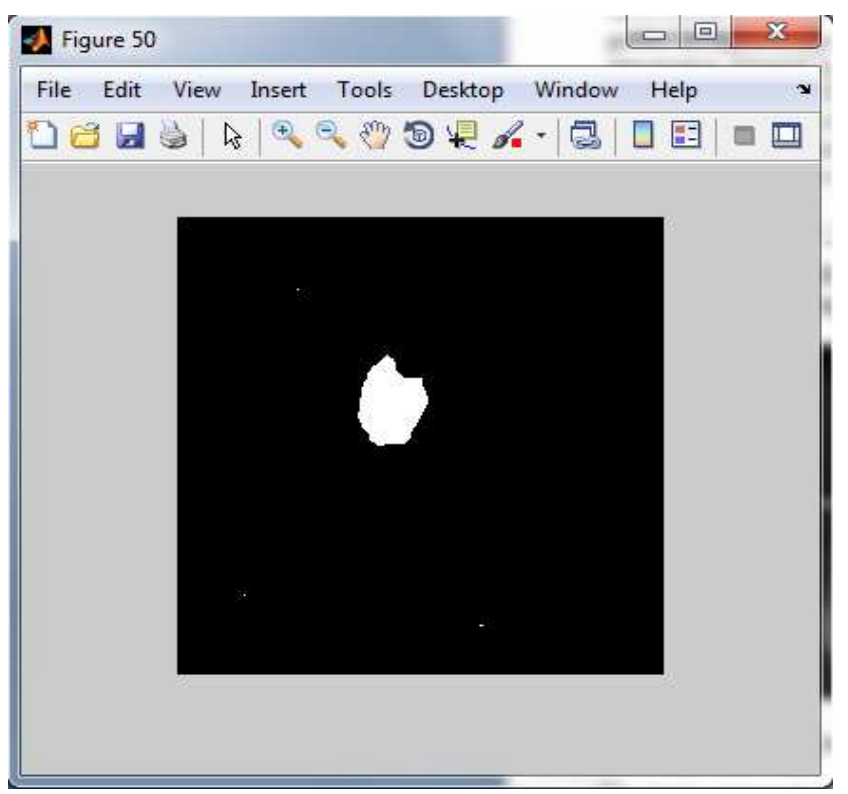

Fig -5: Tumor detected

\section{CONCLUSIONS}

Segmentation of brain image is imperative in surgical planning and treatment planning in the field of medicine. In this work, we have proposed a computer aided system for brain MR image segmentation for detection of tumor location using $\mathrm{K}$ - means clustering algorithm followed by morphological filtering. We were able to segment tumor from different brain MRI images from our database.

\section{REFERENCES}

[1]. Akansha Singh , Krishna Kant Singh, "A Study Of Image Segmentation Algorithms For Different Types Of Images", International Journal of Computer Science Issues, vol. 7,Issue 5, pp 414-417,2010.

[2]. Mansur Rozmin, Prof. Chhaya Suratwala, Prof. Vandana Shah,'Implementation Of Hard C-Means Clustering Algorithm For Medical Image Segmentation", Journal Of Information Knowledge and Research in Electronics and Communication Engineering,vol.2, no.2, pp 436-440,Nov12-Oct13.

[3]. Rajesh Kumar Rai, Trimbak R. Sontakke, "Implementation of Image Denoising using Thresholding Techniques",International Journal of Computer Technology and Electronics Engineering (IJCTEE),vol.1,no. 2, pp 6-10.

[4]. T.Kalaiselvi, S.Vijayalakshmi, K.Somasundara, "Segmentation of Brain Portion from MRI of Head Scans Using Kmeans Cluster", International Journal of Computational Intelligence and Informatics, vol. 1, no. 1, pp 75-79,2011.

[5]. S.S Mankikar , "A Novel Hybrid Approach Using Kmeans Clustering and Threshold filter For Brain Tumor Detection", International Journal of Computer Trends and Technology, vol. 4, no.3, pp 206-209,2013.

[6]. M.C. Jobin Christ, R.M.S.Parvathi, "Segmentation of Medical Image using Clustering and Watershed Algorithms", American Journal of Applied Sciences, vol. 8, pp 1349-1352, 2011.

[7]. Manali Patil, Mrs.Prachi Kshirsagar, Samata Prabhu, Sonal Patil, Sunilka Patil," Brain Tumor Identification Using K-Means Clustering”, International Journal of Engineering Trends and Technology,vol. 4,no. 3,pp 354-357,2013.

[8]. P.Dhanalakshmi , T.Kanimozhi, "Automatic Segmentation of Brain Tumor using K-Means Clustering and its Area Calculation", International Journal of Advanced Electrical and Electronics Engineering ,vol. 2,no. 2,pp 130-134,2013.

[9]. Sanjay Kumar Dubey, Soumi Ghosh, "Comparative Analysis of K-Means and Fuzzy C Means Algorithms", International Journal of Advanced Computer Science and Applications, vol. 4, no. 4, pp 35-39,2013.

[10]. M. Masroor Ahmed, Dzulkifli Bin Mohamad, "Segmentation of Brain MR Images for Tumor Extraction by Combining Kmeans Clustering and Perona-Malik Anisotropic Diffusion Model", International Journal of Image Processing, vol. 2 , no. 1, pp 27-34,2008.

[11]. Anam Mustaqeem, Ali Javed, Tehseen Fatima, "An Efficient Brain Tumor Detection Algorithm Using Watershed \& Thresholding Based Segmentation", I.J. Image, Graphics and Signal Processing, vol. 10,no. 5, pp 34-39,2012. 
[12]. P.Vasuda, S.Satheesh, "Improved Fuzzy C-Means Algorithm for MR Brain Image Segmentation", International Journal on Computer Science and Engineering (IJCSE), vol. 02, no.05, pp 1713-1715, 2010.

[13]. Ananda Resmi S, Tessamma Thomas, "Automatic Segmentation Framework for Primary Tumors from Brain MRIs Using Morphological Filtering Techniques", in $5^{\text {th }}$ Int Conf on Biomedical Engineering and Informatics, 2012,IEEE

[14]. Sindhushree. K. S, Mrs. Manjula. T. R, K. Ramesha, Detection And 3d Reconstruction Of Brain Tumor From Brain Mri Images, International Journal of Engineering Research \& Technology (IJERT), vol. 2, no. 8, pp 528-534, 2013.

[15]. Chang Wen Chen, Jiebo Luo, Kevin J. Parker,"Image Segmentation via Adaptive K-Mean Clustering and Knowledge-Based Morphological Operations with Biomedical Applications", IEEE Trans. Image Process.,vol.7,no.12, pp1673-1683, 1998.

[16]. J.Selvakumar, A.Lakshmi, T.Arivoli,"Brain Tumor Segmentation And Its Area Calculation In Brain MR Images Using K-Mean Clustering And Fuzzy C -Mean Algorithm", Int Conf On Advances In Engineering, Science and Management (ICAESM),2012,IEEE.

[17]. J.Vijay, J.Subhashini, "An Efficient Brain Tumor Detection Methodology Using K-Means Clustering Algorithm", in Int Conf on Communication and Signal Processing, 2013, IEEE.

[18]. G.Evelin Sujji, Y.V.S Lakshmi, G.Wiselin Jiji,"MRI Brain Image Segmentation based on Thresholding", International Journal of Advanced Computer Research, vol.3 no.1, pp 97-101, 2013.

[19]. Ms.Pritee Gupta, Ms Mrinalini Shringirishi, Dr.Yashpal Singh,'Implementation of Brain Tumor Segmentation in brain MR Images using K Means Clustering and Fuzzy C-Means Algorithm", International Journal of Computers \& Technology,vol.5.no.1, pp 54-59, May-June 2013.

[20]. S.M. Ali, Loay Kadom Abood, and Rabab Saadoon Abdoon,"Brain Tumor Extraction in MRI images using Clustering and Morphological Operations Techniques", International Journal of Geographical Information System Applications and Remote Sensing,vol.4,no.1,2013.

[21]. Pratibha Sharma, Manoj Diwakar, Sangam Choudhary, "Application of Edge Detection for Brain Tumor Detection", International Journal of Computer Applications, vol.58, no.16, pp 21-25, 2012.

\section{BIOGRAPHIES}

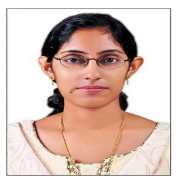

Rohini Paul Joseph received her B.Tech in Electronics and Biomedical Engineering from Govt. Model Engineering College, Cochin University of Science and Technology, Ernakulam. Currently pursuing M.Tech in VLSI and Embedded Systems, at Toc H Institute of Science and Technology, Cochin University of Science and Technology, Ernakulam. Her area of interests include VLSI, Medical Image Processing.

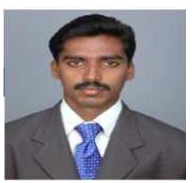

C. Senthil Singh received the B.E. degree in Electrical and Electronics Engineering from the MS University, India, M.Tech in VLSI Design. Currently doing Ph.D. in Information and Communication in Anna University, Chennai, India. His research interest includes VLSI, Image Processing. 